\title{
Expression of 4-hydroxynonenal in esophageal squamous cell carcinoma
}

\author{
YING ZHANG $^{1 *}, \mathrm{HU} \mathrm{WANG}^{2^{*}}, \mathrm{KEZHOU} \mathrm{WU}^{2}$ and ZHAOYONG $\mathrm{LIU}^{2}$ \\ ${ }^{1}$ Department of Pathology, Shantou University Medical College; ${ }^{2}$ Department of Orthopaedic Oncology, \\ First Affiliated Hospital of Shantou University Medical College, Shantou, Guangdong 515041, P.R. China
}

Received October 30, 2015; Accepted January 10, 2017

DOI: $10.3892 / \mathrm{ol} .2017 .6127$

\begin{abstract}
Esophageal squamous cell carcinoma (ESCC) is one of the most common types of cancer in Asia, particular in China. However, the pathogenesis of ESCC has not previously been well demonstrated. A major product of lipid peroxidation, 4-hydroxynonenal (4-HNE), is considered to be an oxidative stress inducer, as it is involved in the pathogenesis of a number of degenerative diseases, including Alzheimer's disease, atherosclerosis, cataracts and cancer. In order to investigate the association between oxidative stress and the pathogenic process of ESCC, the present study determined the expression levels of 4-HNE in 23 non-malignant esophageal epithelial tissues, 11 esophageal carcinoma in situ tissues and 57 ESCC tissues from patients in the Chaoshan area, a high-risk region for esophageal cancer in China. A significantly higher expression level of 4-HNE was identified in ESCC tissues compared with that in non-malignant esophageal epithelial tissues $(\mathrm{P}<0.05)$. Furthermore, immunohistochemical analysis demonstrated that expression levels of 4-HNE were significantly associated with the clinical stage. The patients with positive staining of 4-HNE revealed a poorer clinical outcome compared with that of patients with negative staining. 4-HNE was significantly associated with the severity of inflammation and increased with the progression of precancerous lesions $(\mathrm{P}<0.05)$. These results provide pathological evidence that oxidative stress is a driving force of ESCC carcinogenesis.
\end{abstract}

\section{Introduction}

Esophageal cancer typically occurs in the middle thoracic of the esophagus and is one of the leading causes of cancer-associated

Correspondence to: Dr Zhaoyong Liu, Department of Orthopaedic Oncology, First Affiliated Hospital of Shantou University Medical College, 57 Changping Road, Shantou, Guangdong 515041, P.R. China E-mail: langtian07@hotmail.com

\section{${ }^{*}$ Contributed equally}

Key words: 4-hydroxynonenal, esophageal squamous cell carcinoma, prognostic, oxidative stress, carcinogenesis mortality in China (1). There are two distinct types of esophageal cancer, esophageal squamous cell carcinoma (ESCC), which may occur along the entire esophagus, and esophageal adenocarcinoma, which may develop due to short-segment gastro-esophageal reflux disease (2). However, the mechanisms underlying the development of ESCC have previously not been well demonstrated. The Chaoshan region has one of the highest incidences of esophageal cancer in China, and the age-standardized incidence rates in the Chaoshan area for ESCC were 74/100,000, which is $~ 10$ times greater than the worldwide rate in 2014 (3-5). These findings indicate that there is a unique environment and/or genetic factors involved in the pathogenesis of ESCC.

Oxidative stress is an imbalance between the production of reactive oxygen species (ROS) and antioxidant defenses (6). The major mechanisms underlying the oxidative stress-mediated promotion of cancer have been previously demonstrated $(7,8)$. A certain mechanism involved oxidative stress-damaged DNA and led to cell transformation, which promoted cancer development (8). Another mechanism was induced oxidative stress, which activated numerous cell signaling pathways and may promote carcinogenesis (8). However, there are specific mechanisms that require further investigation. Chronic inflammation is another important risk factor associated with a number of human cancer subtypes, including colon adenocarcinoma and pancreatic cancer $(9,10)$. The association between inflammation and cancer has been clearly demonstrated in previous studies $(6,9,10)$. However, further pathological evidence is required in order to support the results of these studies.

The present study investigated the hypothesis that oxidative stress is one of the driving forces of ESCC. The expression of 4-hydroxynonenal (4-HNE) in a large cohort of ESCC tissues obtained from patients living in Chaoshan was evaluated by immunohistochemistry (IHC) in order to determine the clinical and prognostic significance of 4-HNE in ESCC. Finally, the association of 4-HNE expression level and severity of inflammation in ESCC was determined.

\section{Materials and methods}

ESCC tumor samples. The present study obtained 57 consecutive ESCC tumors from surgery, randomly selected from the First Affiliated Hospital of Shantou University Medical College 
(Shantou, China) from July 2000 to June 2012. All patients underwent potentially curative surgery without preoperative chemotherapy or radiotherapy. In this cohort, 46 patients were male and 11 were female; the age range was 40-75 years, with a median of 58 years. Follow-up data were available for all patients. The majority of patients $(48,84.2 \%)$ succumbed to the disease during the follow-up period (median, 33.6 months). The present study was approved by the Ethics Committee of the Medical College of Shantou University (Shantou, China). Written informed consent was obtained from all patients prior to enrollment in the present study.

IHC. IHC staining was performed using the EnVision Labeled Peroxidase System (Dako; Agilent Technologies, Inc., Santa Clara, CA, USA), according to manufacturer's protocol. Consecutive sections of tissue ( $4-\mu \mathrm{m}$ thick) were obtained from each sample and deparaffinized in dimethyl benzene, rehydrated through a graded ethanol series and incubated with fresh $3 \% \mathrm{H}_{2} \mathrm{O}_{2}$ for $10 \mathrm{~min}$ in order to inhibit endogenous peroxidase activity. The tissues were subsequently washed in PBS, and antigen retrieval was performed by microwave heating at $100^{\circ} \mathrm{C}$ for $20 \mathrm{~min}$. Following incubation with $10 \mathrm{mmol} / \mathrm{l}$ citrate buffer (pH 6.0) for $20 \mathrm{~min}$, primary antibodies against 4-HNE (\#ab48506; dilution, 1:75; Abcam, Cambridge, UK) were incubated at $4^{\circ} \mathrm{C}$ overnight. Subsequent to washing in PBS three times, immunostaining was visualized with a labeled streptavidin-biotin method using the Dako REAL ${ }^{\mathrm{TM}}$ EnVision $^{\mathrm{TM}}$ Detection system, Peroxidase/3,3'-diaminobenzidine+, Rabbit/Mouse (1:200; catalog no. K5007; Dako; Agilent Technologies, Inc.) and included 20 min incubation with the biotinylated antibody and $20 \mathrm{~min}$ in streptavidin horseradish peroxidase, followed by counterstaining with $10 \%$ hematoxylin, all performed at room temperature. Images were captured using an IM50 microscope (Leica Microsystems GmbH, Wetzlar, Germany) at x400 magnification. IHC staining was evaluated by two pathologists who were blinded to the clinical outcome, and concordance between the two pathologies was guaranteed.

For the analysis of 4-HNE immunostaining, the intensity and percentage of immunostained cells was determined, and scoring of 4-HNE immunostaining was performed. The percentage of positive cells in each case was categorized as: $0(<5 \%$ positive cells), $1+(6-25 \%$ positive cells $), 2+(26-50 \%$ positive cells), $3+(51-75 \%$ positive cells $)$ or $4+(>75 \%$ positive cells). The staining intensity was categorized as $0-3+$ as follows: $3+$, intense positive stain; $2+$, moderate positive stain; $1+$, weak positive stain; 0 , negative stain. The final scores were based on the multiplication of the percentage score and the intensity score, which ranged from 0 to 12 . Tumors were considered to have low 4-HNE expression levels when they were assigned a score of $0-4$, whereas tumors were considered to have high 4-HNE expression levels if they had a final score of $\geq 6$.

Statistical analysis. Data are expressed as the mean \pm standard deviation. The prognostic significance of the expression of various markers was analyzed using the Kaplan-Meier test. The association between 4-HNE and clinical parameters was evaluated using the $\chi^{2}$ or Student's t-test. $P \leq 0.05$ was considered to indicate a statistically significant difference.

\section{Results}

Expression levels of 4-HNE in ESCC patient tissue samples. In order to determine the role of oxidative stress in esophageal tissues, the present study investigated the status of oxidative stress in esophageal epithelial tissues exhibiting various degrees of chronic inflammation. For identifying oxidative stress, 4-HNE is one of most bioactive and studied lipid peroxidation biomarkers (11). For the evaluation of the expression levels of 4-HNE in the cohort of ESCC tissue samples, the present study performed IHC on paraffin-embedded tissues. The immunoreactivity of 4-HNE was determined based on the presence of cytoplasmic staining, and was identified in the majority of ESCC tissue samples (40/57, 70.1\%; Fig. 1A, a and b). The staining was categorized as negative $(\mathrm{n}=17,29.8 \%)$ if the staining score was 0 , and positive if the staining score was $>0(n=40,70.2 \%)$. Furthermore, the expression level of 4-HNE was positive in $8 / 23$ (34.8\%) benign esophageal epithelial tissue samples. The remaining 15/23 (65.2\%) tissue samples displayed no detectable cytoplasmic expression level of 4-HNE (Fig. 1A, c and d). The esophageal carcinoma in situ tissues revealed relatively negative 4-HNE immunostaining in 6/11 cases (54.5\%) and positive staining in $5 / 11$ cases (45.5\%; Fig. $1 \mathrm{~A}$, e and f). The IHC score of 4-HNE is presented in Fig. 1B. The final IHC score of ESCC tissues is significantly higher compared with that of benign esophageal epithelial tissues $(\mathrm{P}<0.05)$; however, the score of 4-HNE in carcinoma in situ tissue samples is higher compared with that in benign tissues, but no significant difference was identified $(\mathrm{P}=0.067)$.

Clinical significance of 4-HNE expression level in ESCC tissues. The present study investigated whether the expression level of 4-HNE determined by IHC was associated with various clinicopathological parameters, including gender, location and size of the tumor, lymph node metastasis, histologic grade, depth of tumor invasion and overall clinical stage. As summarized in Table I, the results revealed that the expression level of 4-HNE was associated with the clinical stage $(\mathrm{P}=0.043)$. Tissue samples with positive 4-HNE expression levels also exhibited an association with a late degree of clinical stage. The expression level of 4-HNE revealed no significant association with other clinicopathological parameters.

Clinical follow-up data were available for all patients included in the present study (median follow-up time, 33.6 months; range, 1-68 months). The survival data was analyzed by the Kaplan-Meier test. The overall survival time of patients with 4-HNE-positive tumors was significantly longer compared with that of patients with 4-HNE-negative tumors (30.7 vs. 10.9 months; $\mathrm{P}=0.029$; Fig. 2).

Expression level of 4-HNE is associated with the severity of chronic inflammation in ESCC tissues. Oxidative stress has previously been reported to be an important factor in the promotion of ESCC carcinogenesis (12). Therefore, the present study hypothesized that the expression level of 4-HNE was associated with the stage of ESCC chronic inflammation of ESCC tissue samples. In order to support this hypothesis, the present study evaluated the status of chronic inflammation in esophageal tissues and revealed that chronic inflammation was positively associated with the level of dysplasia in esophageal 
A

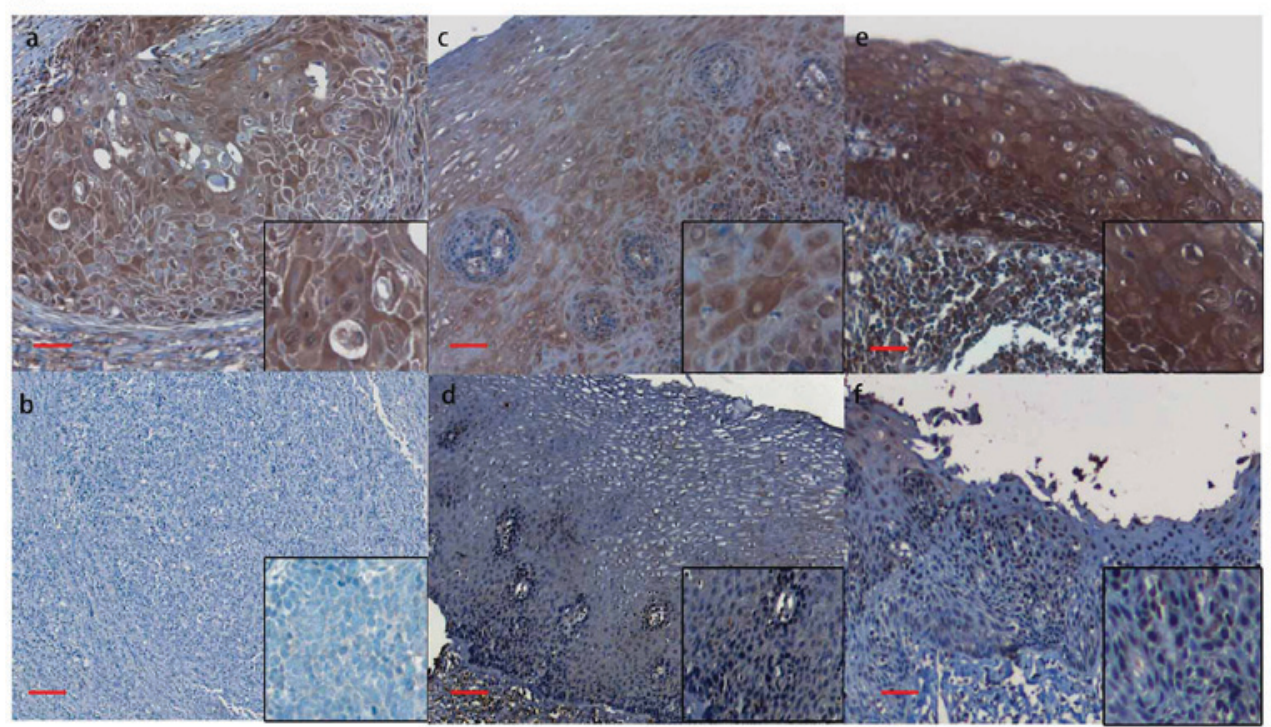

B

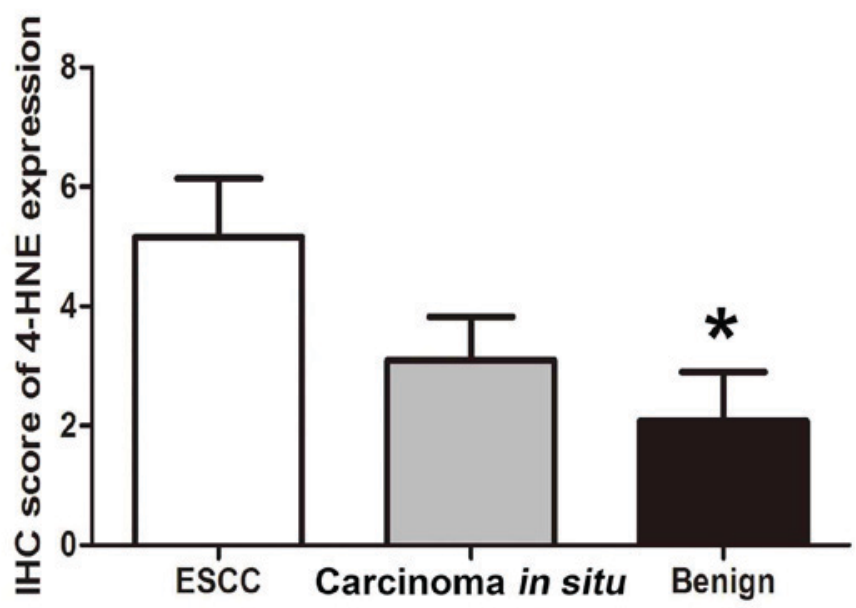

Figure 1. Heterogeneous 4-HNE expression level in ESCC. (A) IHC analysis was performed on formalin-fixed paraffin-embedded tissues, and the expression levels of 4-HNE were investigated in the cytoplasm. Based on the staining, tumors in the cohort were categorized into (a) 4-HNE-positive or (b) 4-HNE-negative in ESCC tissues; the normal epithelium was categorized into (c) 4-HNE-positive or (d) 4-HNE-negative; carcinoma in situ was categorized into (e) 4-HNE-positive or (f) 4-HNE-negative. (B) IHC score of 4-HNE expression in ESCC tissues in situ and benign tissues. IHC staining scale bar, 50 $\mu \mathrm{m}$. "P<0.05. 4-HNE, 4-hydroxynonenal; ESCC, esophageal squamous cell carcinoma; IHC, immunohistochemistry.

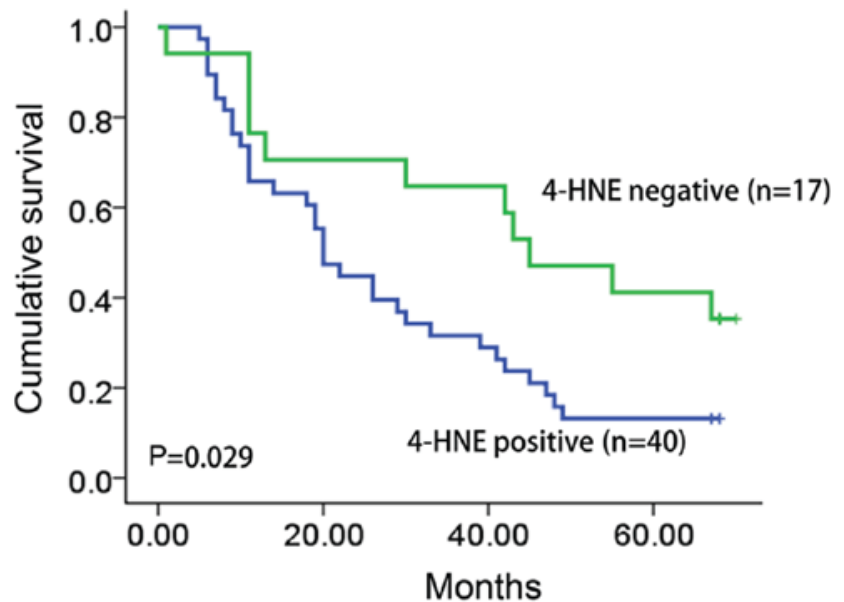

Figure 2. Kaplan-Meier analysis of 4-HNE expression level in esophageal squamous cell carcinoma patient samples. By performing the Kaplan-Meier test, the present study revealed a significant association between overall survival and the expression level of $4-\mathrm{HNE}(\mathrm{P}=0.029)$, when the two groups were defined as positive and negative. 4-HNE, 4-hydroxynonenal. epithelial tissues, indicating that inflammation is a potential factor promoting esophageal carcinogenesis (Fig. 3A).

The present study determined the association of 4-HNE with the inflammation level in ESCC tissues. Based on the severity of chronic inflammation, 57 ESCC tissue samples were divided into negative, mild and severe subgroups. An increased level of staining of 4-HNE was identified in esophageal tissues that had an increased level of chronic inflammation (Fig. 3B). The strongest immunostaining of 4-HNE was observed in severe dysplastic tissues accompanied with severe chronic inflammation (Fig. 3C), indicating that oxidative stress is a potential factor promoting ESCC inflammation.

\section{Discussion}

The present study evaluated the expression levels of 4-HNE in a cohort of ESCC patients and demonstrated that 4-HNE expression is significantly higher in ESCC tissues, compared with that in benign esophageal epithelial tissues. Furthermore, the 


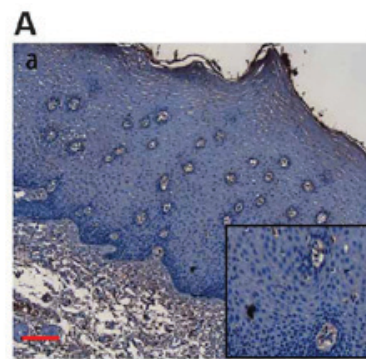

Normal esophageal epithelial

B

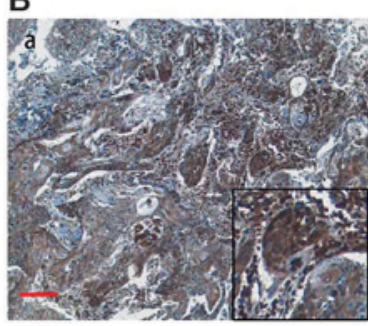

Severe inflammation

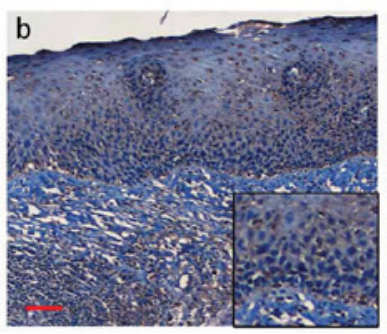

Carcinoma in situ with inflammation

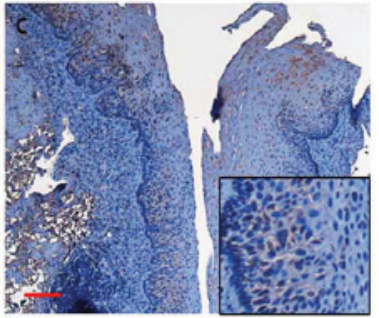

Normal esophageal epithelial with/without inflammation

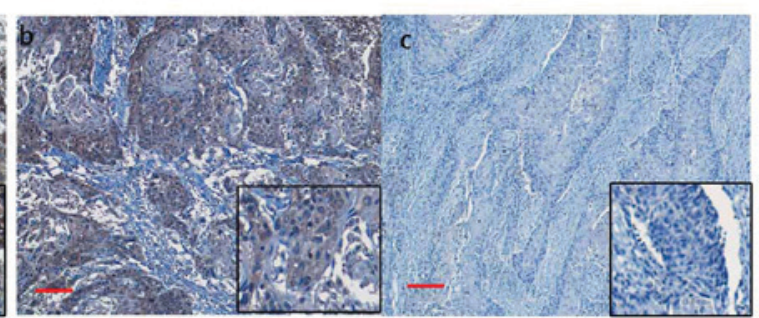

Mild inflammation

Normal

C

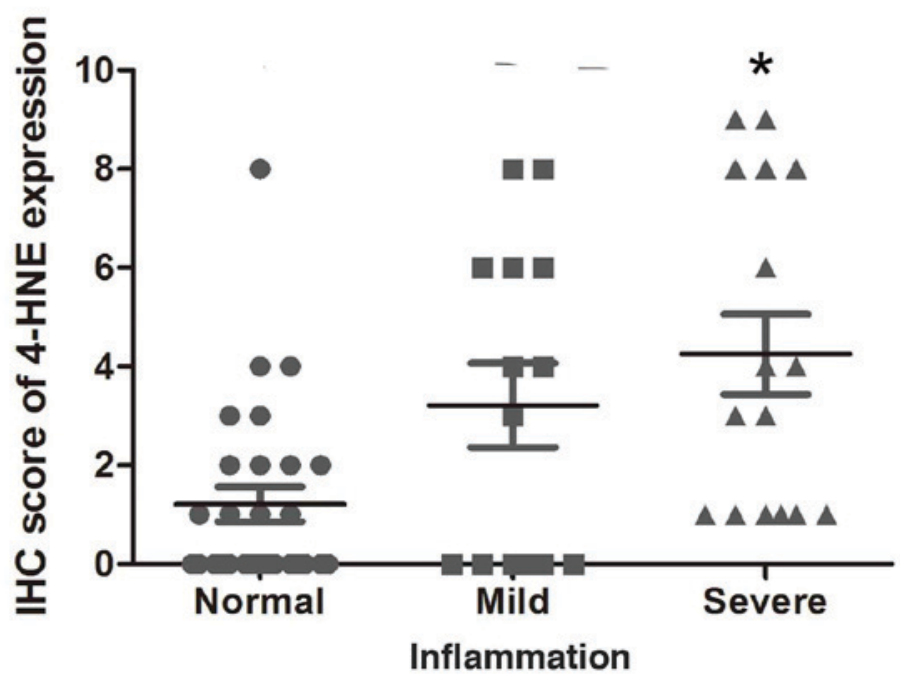

Figure 3. Expression level of 4-HNE in ESCC tissues with a variety of inflammation severities. (A) The expression level of 4-HNE in (a) normal esophageal epithelial is negative and (b) the expression of carcinoma in situ tissues with inflammation is positive for 4-HNE. (c) In the same tissue, the expression level of 4-HNE is higher in epithelial tissues with inflammation compared with that of normal epithelial tissues. (B) Expression level of 4-HNE in ESCC tissues with a variety of inflammation severity. (a) Tissues with severe inflammation demonstrated a high expression level of 4-HNE, and (b) low 4-HNE expression level and (c) negative 4-HNE staining was identified in tissues with no inflammation. (C) The IHC score of 4-HNE was determined in ESCC tissue subgroups with a variety of inflammation severity. The IHC score of $4-\mathrm{HNE}$ in severe inflammation is significantly higher compared with normal tissues. IHC staining scale bar, $50 \mu \mathrm{m}$. ${ }^{*} \mathrm{P}<0.05$.

4-HNE expression level was significantly associated with the poorest clinical stage of ESCC. Patients with positive staining of 4-HNE revealed poorer clinical outcomes compared with patients with a negative 4-HNE expression.

ESCC is one of the most common cancer subtypes in China, particularly in the Chaoshan region (13). The rate of incidence of ESCC in Chaoshan is amongst the highest in China, indicating that there may be certain genetic and/or environmental factors contributing to ESCC carcinogenesis (1). A previous study revealed that tobacco smoking, and consumption of hot drinks and alcohol are established risk factors for ESCC (14). The potential mechanisms underlying these risk factors may induce endogenous metabolic reactions in cells and produce ROS, including superoxide anions $\left(\mathrm{O}_{2}^{-}\right)$, hydroxyl radicals $\left(\mathrm{OH}^{\circ}\right)$ and organic peroxides (15). ROS serve various roles in cellular processes, and low concentrations of ROS have beneficial effect on cells including the promotion of wound healing and tissue repair, in addition to pathogen destruction (16).

However, the accumulation of ROS may induce oxidative stress to damaged cells and contribute to inflammation-induced tissue damage, carcinogenesis, diabetes, cardiovascular disease, Alzheimer's disease, Parkinson's disease and autoimmune diseases (17). Furthermore, the association between oxidative stress and inflammation has previously been demonstrated in numerous studies $(9,10)$. A previous study demonstrated that oxidative stress induces a number of diseases, including chronic inflammation and various cancer subtypes (9). Oxidative stress may induce DNA damage and increase the mutation rate of cells, and promote cell transformation (8). However, oxidative stress may also activate certain cell signaling pathways and 
Table I. Association of 4-HNE expression level with various clinicopathological parameters in ESCC.

4-HNE expression level determined by IHC

Parameter No. of cases Negative Positive P-value

Age (years)

$$
\leq 57
$$

$>58$ 28 29

6
11

22

0.248

Gender

Male
Female

46

11

12
5

34

0.275

Tumor site

Upper
Middle

6
45
6

$\begin{array}{rr}4 & 2 \\ 23 & 22 \\ 3 & 3\end{array}$

Lower

6

Differentiation

Poor
Intermediate

4
31
22

$\begin{array}{rr}3 & 1 \\ 15 & 16 \\ 10 & 12\end{array}$

Tumor size $(\mathrm{cm})$

$\geq 5$
$<5$

38

13
4

Depth of invasion

$\begin{array}{lrrrr}\text { T1-T2 } & 48 & 13 & 35 & 0.428 \\ \text { T3-T4 } & 9 & 4 & 5 & \\ \text { Lymph metastasis } & & & & \\ \text { Yes } & 30 & 8 & 22 & 0.773 \\ \text { No } & 27 & 9 & 18 & \end{array}$

Clinical stage

$\begin{array}{rrrrr}1 & 2 & 1 & 1 & 0.043 \\ 2 & 26 & 18 & 8 & \\ 3 & 27 & 8 & 19 & \\ 4 & 2 & 1 & 1 & \end{array}$

4-HNE, 4-hydroxynonenal; ESCC, esophageal squamous cell carcinoma; IHC, immunohistochemistry.

contribute to cell survival, angiogenesis and metastasis (9). Oxidative stress may be involved in various phases of tumorigenesis, including cell survival, proliferation, invasion and chemoresistance (9). 4-HNE is considered to be a product of lipid peroxidation and an inducer of oxidative stress (12).

4-HNE is a major product of lipid peroxidation that forms covalent adducts with nucleophilic functional groups in macromolecules, including proteins, DNA and lipids (18). In previous studies, 4-HNE was identified to be involved in a number of degenerative diseases, including Alzheimer's disease, atherosclerosis and cancer (19-21). For example, the expression level of 4-HNE was increased in the blood and muscles of obese patients, compared with patients of a healthy weight $(22,23)$. However, the expression level of 4-HNE has not been extensively investigated in cancer tissues. The present study demonstrated that the expression level of 4-HNE is higher in ESCC tissues compared with that in benign esophageal epithelial and esophageal carcinoma in situ tissues, indicating that oxidative stress may be a driving force of ESCC carcinogenesis. In conclusion, the results of the present study suggested that 4-HNE may be highly expressed in ESCC tissues and that a high expression level of 4-HNE was associated with the severity of inflammation in ESCC. The present study provided evidence that oxidative stress may promote the tumorigenicity and inflammation of ESCC tissues.

\section{References}

1. Su M, Liu M, Tian DP, Li XY, Zhang GH, Yang HL, Fan X, Huang $\mathrm{HH}$ and Gao YX: Temporal trends of esophageal cancer during 1995-2004 in Nanao Island, an extremely high-risk area in China. Eur J Epidemiol 22: 43-48, 2007.

2. Napier KJ, Scheerer M and Misra S: Esophageal cancer: A review of epidemiology, pathogenesis, staging workup and treatment modalities. World J Gastrointest Oncol 6: 112-120, 2014.

3. Tang WR, Chen ZJ, Lin K, Su M and Au WW: Development of esophageal cancer in Chaoshan region, China: Association with environmental, genetic and cultural factors. Int J Hyg Environ Health 218: 12-18, 2015.

4. Li K and Yu P: Food groups and risk of esophageal cancer in Chaoshan region of China: A high-risk area of esophageal cancer. Cancer Invest 21: 237-240, 2003.

5. Liu S, Huang B, Huang H, Li X, Chen G, Zhang G, Lin W, Guo D, Wang J, Yu Z, et al: Patrilineal background of esophageal cancer and gastric cardia cancer patients in a Chaoshan high-risk area in China. PLoS One 8: e81670, 2013.

6. Reuter S, Gupta SC, Chaturvedi MM and Aggarwal BB: Oxidative stress, inflammation, and cancer: How are they linked? Free Radic Biol Med 49: 1603-1616, 2010.

7. Sosa V, Moliné T, Somoza R, Paciucci R, Kondoh H and LLeonart ME: Oxidative stress and cancer: An overview. Ageing Res Rev 12: 376-390, 2013.

8. Georgakilas AG: Oxidative stress, DNA damage and repair in carcinogenesis: Have we established a connection? Cancer Lett 327: 3-4, 2012.

9. Shacter E and Weitzman SA: Chronic inflammation and cancer. Oncology (Williston Park) 16: 217-226, 229; discussion 230-232, 2002.

10. Rakoff-Nahoum S: Why cancer and inflammation? Yale J Biol Med 79: 123-130, 2006.

11. Chen ZH and Niki E: 4-hydroxynonenal (4-HNE) has been widely accepted as an inducer of oxidative stress. Is this the whole truth about it or can 4-HNE also exert protective effects? IUBMB Life 58: 372-373, 2006.

12. Sehitogullar A, Aslan M, Sayr F, Kahraman A and Demir H: Serum paraoxonase-1 enzyme activities and oxidative stress levels in patients with esophageal squamous cell carcinoma. Redox Rep 9: 199-205, 2014.

13. Zhang Y, Zhang Y, Yun H, Lai R and Su M: Correlation of STAT1 with apoptosis and cell-cycle markers in esophageal squamous cell carcinoma. PLoS One 9: e113928, 2014.

14. Islami F, Cao Y, Kamangar F, Nasrollahzadeh D, Marjani HA, Shakeri R, Fahimi S, Sotoudeh M, Dawsey SM, Abnet CC, et al: Reproductive factors and risk of esophageal squamous cell carcinoma in northern Iran: A case-control study in a high-risk area and literature review. Eur J Cancer Prev 22: 461-466, 2013.

15. Hardbower DM, de Sablet T, Chaturvedi R and Wilson KT: Chronic inflammation and oxidative stress: The smoking gun for helicobacter pylori-induced gastric cancer? Gut Microbes 4: 475-481, 2013.

16. Ray PD, Huang BW and Tsuji Y: Reactive oxygen species (ROS) homeostasis and redox regulation in cellular signaling. Cell Signal 24: 981-990, 2012.

17. Uttara B, Singh AV,Zamboni P and Mahajan RT: Oxidative stress and neurodegenerative diseases: A review of upstream and downstream antioxidanttherapeutic options. Curr Neuropharmacol 7: 65-74, 2009.

18. Dalleau S, Baradat M, Guéraud F and Huc L: Cell death and diseases related to oxidative stress: 4-hydroxynonenal (HNE) in the balance. Cell Death Differ 20: 1615-1630, 2013. 
19. Siegel SJ, Bieschke J, Powers ET and Kelly JW: The oxidative stress metabolite 4-hydroxynonenal promotes Alzheimer protofibril formation. Biochemistry 46: 1503-1510, 2007.

20. LeonarduzziG,Chiarpotto E,Biasi FandPoli G:4-Hydroxynonenal and cholesterol oxidation products in atherosclerosis. Mol Nutr Food Res 49: 1044-1049, 2005.

21. Zhong $\mathrm{H}$ and $\mathrm{Y}$ in $\mathrm{H}$ : Role of lipid peroxidation derived 4-hydroxynonenal (4-HNE) in cancer: Focusing on mitochondria. Redox Biol 4: 193-199, 2015.
22. Mattson MP: Roles of the lipid peroxidation product 4-hydroxynonenal in obesity, the metabolic syndrome, andassociated vascular and neurodegenerative disorders. Exp Gerontol 44: 625-633, 2009.

23. Samjoo IA, Safdar A, Hamadeh MJ, Raha S and Tarnopolsky MA: The effect of endurance exercise on both skeletal muscle and systemic oxidative stress in previously sedentary obese men. Nutr Diabetes 3: e88, 2013. 